ESAIM: M2AN 46 (2012) 929-947

DOI: $10.1051 / \mathrm{m} 2 \mathrm{an} / 2011068$
ESAIM: Mathematical Modelling and Numerical Analysis

www.esaim-m2an.org

\title{
EFFECT OF THE POLARIZATION DRIFT IN A STRONGLY MAGNETIZED PLASMA
}

\author{
DANIEL HAN-KWAN ${ }^{1}$
}

\begin{abstract}
We consider a strongly magnetized plasma described by a Vlasov-Poisson system with a large external magnetic field. The finite Larmor radius scaling allows to describe its behaviour at very fine scales. We give a new interpretation of the asymptotic equations obtained by Frénod and Sonnendrücker [SIAM J. Math. Anal. 32 (2001) 1227-1247] when the intensity of the magnetic field goes to infinity. We introduce the so-called polarization drift and show that its contribution is not negligible in the limit, contrary to what is usually said. This is due to the non linear coupling between the Vlasov and Poisson equations.
\end{abstract}

Mathematics Subject Classification. 35Q83, 76X05, 82D10.

Received March 8, 2011.

Published online February 3, 2012.

\section{INTRODUCTION}

\subsection{The finite Larmor radius scaling for the Vlasov-Poisson equation}

Consider a hot plasma made of negatively charged particles (electrons) and positively charged particles (ions). For simplicity, we make the hypothesis that there is only one species of ions. We assume that the temperature is so high that collisions can be neglected. Then, the motion of a charged particle (of mass $m$ and charge $q$ ) is described by the Newton equations:

$$
\left\{\begin{array}{l}
\frac{\mathrm{d} X}{\mathrm{~d} t}=V, \\
\frac{\mathrm{d} V}{\mathrm{~d} t}=\frac{q}{m}(E(t, x)+V \wedge B(t, x)),
\end{array}\right.
$$

where $X$ denotes the position and $V$ the velocity of the particle. The fields $E$ and $B$ are respectively the electric and magnetic fields, which are created collectively by the charged particles themselves. Let $T$ and $L$ be the characteristic time and length of observation. Let $c$ be the speed of light. We assume that:

$$
\frac{L}{T c} \gg 1
$$

Keywords and phrases. Vlasov-Poisson equation, strong magnetic field regime, finite Larmor radius scaling, electric drift, polarization drift, oscillations in time.

1 École Normale Supérieure, Département de Mathématiques et Applications, 45 rue d’Ulm, 75230 Paris Cedex 05 France. daniel.han-kwan@ens.fr 
in which case the electrostatic approximation is relevant: this means that we can consider a Poisson equation instead of the full Maxwell equations, in order to determine the electric field created by the plasma, and assume that the magnetic field is an exterior stationary field that satisfies the divergence free condition:

$$
\operatorname{div}_{x} B=0 .
$$

For simplicity we restrict to the most simple geometric case where the magnetic field has constant direction and modulus. An orthonormal basis $\left(e_{1}, e_{2}, e_{3}\right)$ of $\mathbb{R}^{3}$ being fixed, we set:

$$
B(x)=\bar{B} e_{3},
$$

with $\bar{B}$ constant and uniform. For any vector $A=\left(A_{1}, A_{2}, A_{3}\right)$, we denote $A^{\perp}=\left(A_{2},-A_{1}, 0\right)$ and therefore we can write $v \wedge B(x)=\bar{B} v^{\perp}$.

In this work, in order to describe the plasma, we adopt a kinetic point of view, which means that we do not solve Newton equations for all the particles but rather give a statistical description of their motion. For simplicity, we assume that the particles evolve in the periodic torus $\mathbb{T}^{3}$, which allows to confine them without having to deal with boundary effects. Given one type of charged particles, we introduce the so-called distribution function $f(t, x, v)$, which describes their statistical repartition in the phase space $(x, v)$. The quantity $f(t, x, v) \mathrm{d} x \mathrm{~d} v$ is interpreted as the probability of finding an electron at time $t \in \mathbb{R}^{+}$at position $x \in \mathbb{T}^{3}$ and velocity $v \in \mathbb{R}^{3}$. Then transport is described by the following Vlasov equation:

$$
\left\{\begin{array}{l}
\partial_{t} f+v \cdot \nabla_{x} f+(E+v \wedge B) \cdot \nabla_{v} f=0 \\
f_{t=0}=f_{0}
\end{array}\right.
$$

with $E=-\nabla_{x} V$ and the potential $V$ is computed with a Poisson equation (whose precise form depends on the nature of the particles we have chosen to describe; we will come back to this point soon).

We are particularly interested in strongly magnetized plasmas, that are submitted to an intense magnetic field. Such plasmas are nowadays intensively studied in view of energy production by fusion (we refer to [19] and references therein).

In this paper, we discuss and revisit the finite Larmor radius scaling for the Vlasov-Poisson equation with a strong external magnetic field. This scaling was introduced and first studied in the mathematical literature by Frénod and Sonnendrücker [6].

It is well-known that with a large magnetic field, the typical motion of a charged particle is an helix whose axis has the same direction as the magnetic field. The radius of the helix is usually called the Larmor radius; it vanishes as the intensity of the magnetic field goes to infinity. We refer to Section 2 for some detailed computations. The finite Larmor radius scaling is an anisotropic scaling whose basic principle is to distinguish between the typical observation length in the plane $\left(e_{1}, e_{2}\right)$ (that is orthogonal to the magnetic field) and the typical observation length in the line $\left(e_{3}\right)$ (that is parallel to the magnetic field). The idea is then to consider a perpendicular observation length with the same order as the Larmor radius. This allows to give a description of the behaviour of the plasma at fine scales. This is physically relevant, since it was observed experimentally that the plasma undergoes a "turbulent" behaviour at such scales [14]. Let us also mention that recently, this regime was investigated in $[3,5,9,12,13]$. The scaled Vlasov equation reads ${ }^{2}$ :

$$
\left\{\begin{array}{l}
\partial_{t} f_{\varepsilon}+\frac{v_{\perp}}{\varepsilon} \cdot \nabla_{x_{\perp}} f_{\varepsilon}+v_{\|} \partial_{x_{\|}} f_{\varepsilon}+\left(E_{\varepsilon}+\frac{v^{\perp}}{\varepsilon}\right) \cdot \nabla_{v} f_{\varepsilon}=0 \\
E_{\varepsilon}=-\left(\nabla_{x_{\perp}} V_{\varepsilon}, \varepsilon \partial_{x_{\|}} V_{\varepsilon}\right) \\
f_{\varepsilon, t=0}=f_{0, \varepsilon} .
\end{array}\right.
$$

In this system, $\varepsilon$ is a small positive parameter (roughly, $\varepsilon \sim \frac{1}{\bar{B}}$ ) that we intend to let go to zero, in order to obtain a somehow simplified asymptotic equation.

\footnotetext{
${ }^{2}$ We refer to Appendix A for some details on the scaling.
} 
As mentioned before, this system is coupled with a Poisson equation for the potential $V_{\varepsilon}$, which depends on the nature of the charged particles (ions or electrons) that we intend to describe. The main point is to observe that the mass of an ion is much larger than the mass of an electron, so that their dynamics are completely different.

- If we describe the electrons by the distribution function $f_{\varepsilon}$, we can consider that their mass is of order 1 and that the mass of the ions is equal to $+\infty$. Thus we can assume that the ions are motionless, and for simplicity we assume that their density $n_{i}$ is uniform, equal to 1 . As a result, the Poisson equation in this case reads:

$$
-\varepsilon^{2} \partial_{x_{\|}}^{2} V_{\varepsilon}-\Delta_{x_{\perp}} V_{\varepsilon}=\int f_{\varepsilon} \mathrm{d} v-1
$$

- If we describe the ions by the distribution function $f_{\varepsilon}$, we can consider that their mass is of order 1 and that the mass of the electrons is equal to 0 . Therefore, the electrons instantaneously reach their thermodynamic equilibrium. It is usually assumed that their density $n_{e}$ follows a Maxwell-Boltzmann law, that is:

$$
n_{e}=\mathrm{e}^{V_{\varepsilon}} .
$$

We make the simplifying assumption (commonly done in plasma physics) that this law can be linearized, so that:

$$
n_{e}=1+V_{\varepsilon}
$$

Thus the Poisson equation reads in this case:

$$
V_{\varepsilon}-\varepsilon^{2} \partial_{x_{\|}}^{2} V_{\varepsilon}-\Delta_{x_{\perp}} V_{\varepsilon}=\int f_{\varepsilon} \mathrm{d} v-1
$$

Although (1.5) may seem to be a harmless modification of (1.4), the additional term $V_{\varepsilon}$ actually makes a huge difference in the analysis of the full three-dimensional problem, as it has been discussed in $[12,13]$.

In this paper, we restrict the so-called $2 D$ problem, which means that we restrict to initial data which do not depend on $x_{\|}$and $v_{\|}$. Actually in the $2 D$ setting, considering (1.5) or (1.4) does not make any difference, and thus for simplicity we will describe a gas of electrons and consider the $2 D$ version of (1.4). Then, the scaled Vlasov-Poisson system reads:

$$
\left\{\begin{array}{l}
\partial_{t} f_{\varepsilon}+\frac{v}{\varepsilon} \cdot \nabla_{x} f_{\varepsilon}+\left(E_{\varepsilon}+\frac{v^{\perp}}{\varepsilon}\right) \cdot \nabla_{v} f_{\varepsilon}=0, \quad t \geq 0, x \in \mathbb{T}^{2}, v \in \mathbb{R}^{2} \\
E_{\varepsilon}=-\nabla_{x} V_{\varepsilon} \\
-\Delta_{x} V_{\varepsilon}=\int f_{\varepsilon} \mathrm{d} v-1 \\
f_{\varepsilon, t=0}=f_{0, \varepsilon}
\end{array}\right.
$$

This is the system we will focus on throughout this paper.

\subsection{Short review of the $2 D$ problem and presentation of the issue}

In [6], Frénod et Sonnendrücker showed the following result:

Theorem 1.1 (Frénod-Sonnendrücker). Let $\left(f_{0, \varepsilon}\right)$ a sequence of initial data satisfying the following conditions:

(H1) for any $\varepsilon, f_{0, \varepsilon} \geq 0$ and $\int f_{0, \varepsilon} \mathrm{d} v \mathrm{~d} x=1$;

(H2) there exists $C>0$, for any $\varepsilon, \int f_{0, \varepsilon}|v|^{2} \mathrm{~d} v \mathrm{~d} x \leq C$;

(H3) there exists $q>2$, such that $\left(f_{0, \varepsilon}\right)$ is uniformly bounded in $L^{q}\left(\mathbb{T}^{2} \times \mathbb{R}^{2}\right)$.

We assume that $\left(f_{\varepsilon, 0}\right)$ weakly converges in $L_{x, v}^{2}$ to some $f_{0} \in L_{x, v}^{1} \cap L_{x, v}^{q}$. Let $\left(f_{\varepsilon}\right)$ the global weak solutions in the sense of Arsenev [2] of (1.6) with initial conditions $\left(f_{0, \varepsilon}\right)$. Then, up to a subsequence, $\left(f_{\varepsilon}\right)$ converges in the sense of distributions to $f$ defined by:

$$
f=\int_{0}^{2 \pi} G(t, x+\mathcal{R}(\tau) v, R(\tau) v) \mathrm{d} \tau
$$


with $G$ solution to the equation:

$$
\left\{\begin{array}{l}
\partial_{t} G+\frac{1}{2 \pi}\left(\int_{0}^{2 \pi} \mathcal{R}(\tau) \mathcal{E}(t, \tau, x+\mathcal{R}(-\tau) v) \mathrm{d} \tau\right) \cdot \nabla_{x} G \\
+\frac{1}{2 \pi}\left(\int_{0}^{2 \pi} R(\tau) \mathcal{E}(t, \tau, x+\mathcal{R}(-\tau) v) \mathrm{d} \tau\right) \cdot \nabla_{v} G=0 \\
\mathcal{E}=-\nabla \Phi, \quad-\Delta \Phi=\int G(t, x+\mathcal{R}(\tau) v, R(\tau) v) \mathrm{d} v-1 \\
G_{\mid t=0}=f_{0},
\end{array}\right.
$$

denoting by $R$ and $\mathcal{R}$ the rotation operators defined by

$$
R(\tau)=\left[\begin{array}{cc}
\cos \tau & \sin \tau \\
-\sin \tau & \cos \tau
\end{array}\right], \mathcal{R}(\tau)=(R(\pi / 2)-R(\pi / 2+\tau)) .
$$

One can observe that it is possible to write equation (1.8) in a (slightly) more tractable way, by using the change of variables:

$$
\tilde{G}(t, \tau, x, v):=G\left(t, \tau, x-v^{\perp}, v\right) .
$$

The equation satisfied by $\tilde{G}$ then reads:

$$
\left\{\begin{array}{l}
\partial_{t} \tilde{G}+\frac{1}{2 \pi}\left(\int_{0}^{2 \pi} \mathcal{E}^{\perp}(t, \tau, x-R(\pi / 2-\tau) v) \mathrm{d} \tau\right) \cdot \nabla_{x} \tilde{G} \\
+\frac{1}{2 \pi}\left(\int_{0}^{2 \pi} R(\tau) \mathcal{E}(t, \tau, x-R(\pi / 2-\tau) v) \mathrm{d} \tau\right) \cdot \nabla_{v} \tilde{G}=0 \\
\mathcal{E}=-\nabla \Phi,-\Delta \Phi=\int \tilde{G}(t, x-R(\pi / 2+\tau) v, R(\tau) v) \mathrm{d} v-1 \\
\tilde{G}_{\mid t=0}=f_{0}\left(x-v^{\perp}, v\right)
\end{array}\right.
$$

We can observe that the term:

$$
\frac{1}{2 \pi}\left(\int_{0}^{2 \pi} \mathcal{E}^{\perp}(t, \tau, x-R(-\tau+\pi / 2) v) \mathrm{d} \tau\right) \cdot \nabla_{x}
$$

corresponds to the classical electric drift (we refer to Sect. 2 for some physical insight about the drifts arising in presence of a strong magnetic field):

$$
v_{E}=\frac{E \wedge B}{|B|}=E^{\perp}
$$

in an averaged form (usually called gyro-averaged). This corresponds to an average around a circle of rotation in the orthogonal plane. For any function $F(x)$, we call gyroaverage of $F$ the function:

$$
\langle F\rangle(x, v)=\frac{1}{2 \pi}\left(\int_{0}^{2 \pi} F(x-R(-\tau+\pi / 2) v) \mathrm{d} \tau\right) .
$$

That the gyroaveraged electric drift appears instead of its non-averaged form is related to the finite Larmor radius scaling: any variation within a length of a few Larmor radius is not negligible, and thus this average is natural.

With such an approach, we observe that there is another term, which has a priori no physical meaning:

$$
\frac{1}{2 \pi}\left(\int_{0}^{2 \pi} R(\tau) \mathcal{E}(t, \tau, x-R(-\tau+\pi / 2) v) \mathrm{d} \tau\right) \cdot \nabla_{v} \tilde{G}
$$

The questions we have in mind are the following: what is the physical meaning of this term? Is it possible to make it vanish?

It is interesting to see that the equation used by plasma physicists for numerical simulations, see for instance the GYSELA code ([11]), is a simplified version of this equation, the term (1.13) being neglected (actually there are other drifts, due to geometric effects, but those are not considered here). 
For convenience, in this paper, we will denote $\mathrm{e}^{\mathrm{i} \theta}$ for the vector $\left(\begin{array}{c}\cos \theta \\ \sin \theta\end{array}\right)$.

$$
\left\{\begin{array}{l}
\partial_{t} f+\frac{1}{2 \pi} \int_{0}^{2 \pi} E^{\perp}\left(t, x-|v| \mathrm{e}^{\mathrm{i} \theta+\mathrm{i} \pi / 2}\right) \mathrm{d} \theta \cdot \nabla_{x} f=0, \\
E=-\nabla_{x} V \\
-\Delta_{x} V=\int f\left(t, x-v^{\perp}, v\right) \mathrm{d} v-\int f \mathrm{~d} v \mathrm{~d} x .
\end{array}\right.
$$

Lately there has been some effort to obtain equation (1.14) from (1.6): we refer to the works of Bostan [3], Ghendrih et al. [9] and Frénod and Mouton [5].

In [3], Theorem 5.1, under some well-prepared assumptions on the initial data, the author is able to derive (1.14) from (1.6):

Theorem 1.2 (Bostan). Let $\left(f_{0, \varepsilon}\right)$ a sequence of initial conditions satisfying the following conditions:

(H1) for any $\varepsilon, f_{0, \varepsilon} \geq 0$ and $\int f_{0, \varepsilon} \mathrm{d} v \mathrm{~d} x=1$;

(H2) there exists a bounded nonincreasing function $F_{0} \in L^{\infty}\left(\mathbb{R}^{+}\right) \cap L^{1}\left(\mathbb{R}^{+}, r \mathrm{~d} r\right)$ such that:

$$
f_{0}^{\varepsilon}(x, p) \leq F_{0}(|p|)
$$

(H3) there exists $q>2$, such that $\left(f_{0, \varepsilon}\right)$ is uniformly bounded in $W^{2, q}\left(\mathbb{T}^{2} \times \mathbb{R}^{2}\right)$;

$\left(H_{4}\right)$ there exists a subsequence $\left(\varepsilon_{k}\right)$ such that $\varepsilon_{k} \rightarrow 0$ and a function $f_{0}$ in $W^{2, q}\left(\mathbb{T}^{2} \times \mathbb{R}^{2}\right)$, compactly supported in velocity, and satisfying:

$$
v \cdot \nabla_{x} f_{0}+v^{\perp} f_{0}=0
$$

such that

$$
f_{0, \varepsilon_{k}} \rightarrow f_{0}
$$

strongly in $L^{2}\left(\mathbb{T}^{2} \times \mathbb{R}^{2}\right)$.

Let $\left(f_{\varepsilon_{k}}\right)$ the global weak solutions in the sense of Arsenev [2] of (1.6) with initial conditions $\left(f_{0, \varepsilon_{k}}\right)$. Then there exists $T>0$ such that $\left(f_{\varepsilon_{k}}\right)$ converges strongly in $L^{\infty}(] 0, T\left[, L^{2}\left(\mathbb{T}^{2} \times \mathbb{R}^{2}\right)\right)$ to $f$ solution of $(1.14)$ with initial data $f_{0}$.

It is interesting to compare the assumptions of the two previous theorems. The assumptions (H2) and (H3) in Theorem 1.2 are more demanding than those of Theorem 1.1, the main reason is that one needs much more control on high velocities and also some stronger stability estimates for proving Theorem 1.2. Actually, the most restricting condition corresponds to the assumption (H4): the condition (1.15) means that $f_{0}$ belongs to the kernel of the singular penalization operator $v \cdot \nabla_{x}+v^{\perp} \cdot \nabla_{v}$. This a well-prepared assumption. Unfortunately, in "real life" plasmas, this does not seem relevant, since it is in practice impossible to impose it or even to check that it is satisfied. By opposition, somehow, Theorem 1.1 therefore corresponds to an ill-prepared situation, since there is not such assumption on the initial data.

In [5], the authors also try to derive (1.14) from (1.6) in such an ill-prepared situation. Unfortunately, the goal is reached only by assuming the additional assumption (called "unphysical" in [5]) that $E_{\varepsilon} \rightarrow E$ strongly in time and space.

As a matter of fact, as we shall see afterwards, the electric field $E_{\varepsilon}$ displays oscillations in time of frequency of order $\mathcal{O}(1 / \varepsilon)$. This is due to the nonlinear coupling between the Vlasov and Poisson equations. This means that in general, $E_{\varepsilon}$ never converges strongly, but only weakly. It is well known that weak convergence behaves nastily with respect to multiplication: resonance effects may appear and produce a non-trivial mean transport. As a result, the formal analysis may be false.

In this paper, we consider "general" initial data which do not satisfy the well-prepared assumption. The approach that we carry out allow us to provide a clarification and a better understanding to the gyrokinetic equation (1.8), in particular to the terms (1.13). Our goal is to give evidence that (1.8) actually describes more effects than the mere electric drift, which is the usual interpretation. More specifically, we will exhibit effects 
due to the so-called polarization drift, which is usually considered as a higher order term and thus neglected, in the physics literature. The contribution is due precisely to the fast oscillations in time of the electric field.

This means that if one wants to give an accurate numerical simulation of strongly magnetized plasmas (for instance for industrial purposes), system (1.8) is more relevant than system (1.14).

\subsection{Organization of the paper}

The paper is organized as follows: in Section 2, we first give some physical heuristics in order to understand the expected dynamics for the charged particles. In Section 3, we give and we prove the main results of this paper; our approach is based on a simple non linear change of frame, inspired by the heuristics, which allows to interpret the mysterious terms (1.13) as the effect of the polarization drift (we refer in particular to Thm. 3.10). Finally, we gather in two appendices some elements on the finite Larmor radius scaling and we propose a new physical model that may be more accurate than (1.14).

\section{THE ELECTRIC DRIFT, AND THE POLARIZATION DRIFT}

Let us qualitatively describe the dynamics of a charged particle (of mass $m$ and charge $q$, assumed to be positive for simplicity), under the influence of a given electric field $E$ and a given magnetic field $B$. As before, we restrict to the simplest geometric case: $B$ is uniform and constant. We focus on the dynamics in the perpendicular (to the magnetic field) plane. The main feature of the magnetic field is that it is assumed to be large, so that $|B| \sim \frac{1}{\varepsilon}$, with $\varepsilon \ll 1$ (while $|E| \sim 1$ ). We now perform a multi-scale expansion of the solutions to the Newton equations, that we recall here:

$$
\left\{\begin{array}{l}
\frac{\mathrm{d} X}{\mathrm{~d} t}=V, \\
m \frac{\mathrm{d} V}{\mathrm{~d} t}=q(E+V \wedge B) .
\end{array}\right.
$$

At leading order, the motion is dominated by the influence of the magnetic field and we can neglect the electric field. The equation satisfied by $V$ is then $\frac{\mathrm{d} V}{\mathrm{~d} t}=q V \wedge B$. By some straightforward and elementary computations, we can easily obtain that the motion is circular:

$$
V=R\left(t \Omega_{c}\right) V^{0}, \quad X=X^{0}+r_{L} \mathcal{R}\left(t \Omega_{c}\right) \frac{V^{0}}{\left|V^{0}\right|},
$$

with $R$ and $\mathcal{R}$ the rotation operators defined in (1.9) and we denote by $r_{L}$ the so-called Larmor radius defined by:

$$
r_{L}=\frac{q\left|V^{0}\right|}{m|B|}
$$

and $\Omega_{c}$ the cyclotron frequency:

$$
\Omega_{c}=\frac{q|B|}{m} .
$$

As explained in the introduction, the finite Larmor radius (FLR) scaling consists precisely in considering a typical space length with the same order as $r_{L}$, so that at this scale, neglecting the electric field is not relevant, since higher order effets are no more negligible. This is the matter of the next paragraphs.

Let us first suppose that the electric field $E$ does not depend on time. We reconsider the equation:

$$
m \frac{\mathrm{d} V}{\mathrm{~d} t}=q E+q V \wedge B
$$

and set $\tilde{V}=V-\frac{E \wedge B}{|B|^{2}}$. Then, the velocity field $\tilde{V}$ satisfies the equation:

$$
m \frac{\mathrm{d} \tilde{V}}{\mathrm{~d} t}=q \tilde{V} \wedge B
$$


We thus observe that $\tilde{V}$ thus satisfies the same equation as (2.1) but without electric field. As a consequence, we have:

$$
V_{\perp}=\frac{E \wedge B}{|B|^{2}}+R\left(t \Omega_{c}\right) \tilde{V}_{\perp}^{0}
$$

The motion of the particle is then given by:

$$
X=X^{0}+t \frac{E \wedge B}{|B|^{2}}+\frac{q}{m|B|} \mathcal{R}\left(t \Omega_{c}\right)\left(V_{\perp}^{0}-\frac{E \wedge B}{|B|^{2}}\right) .
$$

We call "electric drift" (also called $E \times B$-drift) the drift:

$$
v_{E}=\frac{E \wedge B}{|B|^{2}} .
$$

We observe that $\left|v_{E}\right| \sim \varepsilon$, and consequently, in the FLR regime, this becomes an effect of order $\mathcal{O}(1)$ (in other words this a leading order term in this regime).

Suppose now that the electric field depends on time (which is the case in most situations). In this case, the equation satisfied by $\tilde{V}$ is the following:

$$
\frac{\mathrm{d} \tilde{V}}{\mathrm{~d} t}=\tilde{V} \wedge B-\frac{1}{|B|^{2}} \frac{\mathrm{d} E}{\mathrm{~d} t} \wedge B
$$

We can set, as in the previous case, $\bar{V}=\tilde{V}+\frac{1}{|B|^{2}} \frac{\mathrm{d} E_{\perp}}{\mathrm{d} t}$. Then the equation satisfied by $\bar{V}$ is the following one:

$$
\frac{\mathrm{d} \tilde{V}}{\mathrm{~d} t}=q \tilde{V} \wedge B-\frac{1}{|B|^{2}} \frac{\mathrm{d}^{2} E_{\perp}}{\mathrm{d} t^{2}} .
$$

We neglect the term $-\frac{1}{|B|^{2}} \frac{\mathrm{d}^{2} E}{\mathrm{~d} t^{2}}$ : indeed, assuming that $\left|\frac{\mathrm{d}^{2} E}{\mathrm{~d} t^{2}}\right|$ is of order 1 , this is seemingly a higher order term in $\varepsilon$, which gives rise to higher order terms.

If we proceed as in the previous case, this results in another drift, called "polarization drift":

$$
v_{p}=\frac{1}{|B|^{2}} \frac{\mathrm{d} E}{\mathrm{~d} t} .
$$

We can notice that if $\left|\frac{\mathrm{d} E}{\mathrm{~d} t}\right|$ is of order 1 , then we have $\left|v_{p}\right| \sim \varepsilon^{2}$. With an observation length of order $\varepsilon$, this is consequently a term of order $\mathcal{O}(\varepsilon)$. As a result, one usually considers that the polarization drift is indeed a higher order term than the electric drift, so it shouldn't have any influence on the asymptotic equation. As first explained in the introduction, we point out that this is likely to be a wrong belief. Indeed, due to some resonance phenomena, there are oscillations in time for the electric field, which entails that $\varepsilon \partial_{t} E_{\varepsilon}$ is actually of order one. Consequently, the scaling analysis is likely to be false and the electric and polarization drifts can be of the same order.

Even worse, the resonance phenomena entail that $-\frac{1}{|B|^{2}} \frac{\mathrm{d}^{2} E}{\mathrm{~d} t^{2}}$ is actually not a higher order term, so it also shouldn't be neglected in order to describe the motion.

\section{EFFECTS of the POLARIZATION DRIFT IN THE FINITE LARMOR RADiUs APPROXIMATION}

\subsection{A dynamical change of frame}

Our goal is now to rigorously justify (in the framework of the kinetic Vlasov-Poisson system) the discussion of the previous section. 
Recall that we a priori expect to obtain a drift $\varepsilon E_{\varepsilon}^{\perp}$. Thus, we consider the new distribution function $f_{\varepsilon}^{\prime}$ defined by the non linear change of frame:

$$
f_{\varepsilon}^{\prime}(t, x, v)=f_{\varepsilon}\left(t, x, v+\varepsilon E_{\varepsilon}^{\perp}\right),
$$

where $f_{\varepsilon}$ satisfies (1.6). We straightforwardly obtain the equation satisfied by $f_{\varepsilon}^{\prime}$ :

$$
\partial_{t} f_{\varepsilon}^{\prime}+\frac{v}{\varepsilon} \cdot \nabla_{x} f_{\varepsilon}^{\prime}+E_{\varepsilon}^{\perp} \cdot \nabla_{x} f_{\varepsilon}^{\prime}+(\underbrace{-\varepsilon \partial_{t} E_{\varepsilon}^{\perp}-\frac{v+\varepsilon E_{\varepsilon}^{\perp}}{\varepsilon} \cdot \nabla_{x}\left(\varepsilon E_{\varepsilon}^{\perp}\right)}_{:=F_{\varepsilon}}) \cdot \nabla_{v} f_{\varepsilon}^{\prime}+\frac{v^{\perp}}{\varepsilon} \cdot \nabla_{v} f_{\varepsilon}^{\prime}=0 .
$$

We call the force $F_{\varepsilon}$ the "polarization force".

Let us now give two approaches (based on filtering techniques) which will help us to let appear the influence of the polarization drift.

1. The first is the approach used in [9]. This one is somehow the easiest, and makes both the electric and the polarization drift in a very clear and explicit way.

Unfortunately, one can not handle the full Vlasov-Poisson system with it, since the oscillations due to magnetic field will remain after the change of variables. Consequently there is a lack of compactness in time if we try to pass to the limit. Nevertheless, this approach will work when the electric field is a chosen external force field.

The change of variables consists of:

$$
g_{\varepsilon}(t, x, v)=f_{\varepsilon}^{\prime}\left(t, x-v^{\perp}, v\right) .
$$

We then obtain the equation satisfied by $g_{\varepsilon}$ :

$$
\begin{aligned}
\partial_{t} g_{\varepsilon} & +\underbrace{E_{\varepsilon}^{\perp}\left(t, x-v^{\perp}\right)}_{\text {electric drift }} \cdot \nabla_{x} g_{\varepsilon}+\underbrace{F_{\varepsilon}^{\perp}\left(t, x-v^{\perp}, v\right)}_{\text {polarization drift }} \cdot \nabla_{x} g_{\varepsilon} \\
& +F_{\varepsilon}\left(t, x-v^{\perp}, v\right) \cdot \nabla_{v} g_{\varepsilon}+\frac{v^{\perp}}{\varepsilon} \cdot \nabla_{v} g_{\varepsilon}=0 .
\end{aligned}
$$

Observe here that $F_{\varepsilon}^{\perp}$ indeed corresponds to the polarization drift defined in (2.2). More explicitly, we have:

$$
F_{\varepsilon}^{\perp}=\varepsilon \partial_{t} E_{\varepsilon}+v \cdot \nabla_{x} E_{\varepsilon}+\varepsilon E_{\varepsilon}^{\perp} \cdot \nabla_{x} E_{\varepsilon} .
$$

2. The second approach is that of [6], that we have evoked in the first section of the paper. It consists of filtering out all the fast time oscillations. We set $h_{\varepsilon}$ defined by:

$$
h_{\varepsilon}(t, x, v)=f_{\varepsilon}^{\prime}(t, x+\mathcal{R}(-t / \varepsilon) v, R(-t / \varepsilon) v),
$$

where $R$ and $\mathcal{R}$ are the rotation operators defined in (1.9).

After some straightforward computations we get:

$$
\begin{aligned}
\partial_{t} h_{\varepsilon} & +E_{\varepsilon}^{\perp}(t, x+\mathcal{R}(-t / \varepsilon) v) \cdot \nabla_{x} h_{\varepsilon}+\mathcal{R}(t / \varepsilon) F_{\varepsilon}(t, x+\mathcal{R}(-t / \varepsilon) v, R(-t / \varepsilon) v) \cdot \nabla_{x} h_{\varepsilon} \\
& +R(t / \varepsilon) F_{\varepsilon}(t, x+\mathcal{R}(-t / \varepsilon) v, R(-t / \varepsilon) v) \cdot \nabla_{v} h_{\varepsilon}=0 .
\end{aligned}
$$

\subsection{External electric field case}

Let us consider the simple case when $E_{\varepsilon}:=E=-\nabla_{x} V$ is an external (smooth) electric field, in $\mathcal{C}_{t, x}^{1} \cap H_{t, x, l o c}^{1}$. In this case, since the electric field does not wildly oscillate, we do not expect to observe any polarization effect in the limit. 
Actually, we recover the same results ${ }^{3}$ as in [9]. It is nevertheless interesting to explain how the contribution of the polarization drift vanishes in our framework.

Theorem 3.1. Let $\left(g_{\varepsilon, 0}\right)$ a sequence of initial data, uniformly bounded in $L_{x, v}^{1} \cap L_{x, v}^{2}$. We assume that $\left(g_{\varepsilon, 0}\right)$ weakly converges in $L_{x, v}^{2}$ to some $g_{0} \in L_{x, v}^{1} \cap L_{x, v}^{2}$. Then up to a subsequence, the sequence $\left(g_{\varepsilon}\right)$ of solutions to (3.3) with initial data $\left(g_{\varepsilon, 0}\right)$ converges in the sense of distributions to $g$ solution to:

$$
\left\{\begin{array}{l}
\partial_{t} g+\frac{1}{2 \pi} \int_{0}^{2 \pi} E^{\perp}\left(t, x-|v| \mathrm{e}^{\mathrm{i} \theta+\mathrm{i} \pi / 2}\right) \mathrm{d} \theta \cdot \nabla_{x} g=0, \\
g_{\mid t=0}=\frac{1}{2 \pi} \int_{0}^{2 \pi} g_{0}\left(x,|v| \mathrm{e}^{\mathrm{i} \theta}\right) \mathrm{d} \theta .
\end{array}\right.
$$

We recall that we use the notation $\mathrm{e}^{\mathrm{i} \theta}=\left(\begin{array}{c}\cos \theta \\ \sin \theta\end{array}\right)$.

Remark 3.2. Until now, we have restricted to the $2 \mathrm{D}$ setting, but in this case the $3 \mathrm{D}$ results would easily follow. Indeed, in the three-dimensional setting we obtain for the polarization force:

$$
F_{\varepsilon}=-\varepsilon \partial_{t} E^{\perp}-v_{\perp} \cdot \nabla_{x_{\perp}} E^{\perp}-\varepsilon E^{\perp} \cdot \nabla_{x_{\perp}} E^{\perp}-\varepsilon v_{\|} \partial_{x_{\|}} E^{\perp} .
$$

The only additional term is the last one, and since $E$ is external it clearly vanishes in the limit. That being said, we restrict to the $2 D$ case for the sake of readability.

Before proving the theorem, we state a technical lemma which explains that due to symmetry reasons, the contributions appearing because of polarization cancel in the limit.

Lemma 3.3. For any $r \geq 0$, we have the cancellations:

$$
\begin{gathered}
\int_{0}^{2 \pi} \mathrm{e}^{\mathrm{i} \theta} \cdot \nabla_{x} E\left(x-r \mathrm{e}^{\mathrm{i} \theta+\mathrm{i} \pi / 2}\right) \mathrm{d} \theta=0, \\
\int_{0}^{2 \pi} \mathrm{e}^{\mathrm{i} \theta} \cdot \nabla_{x} E^{\perp}\left(x-r \mathrm{e}^{\mathrm{i} \theta+\mathrm{i} \pi / 2}\right) \cdot \mathrm{e}^{\mathrm{i} \theta} \mathrm{d} \theta=0 .
\end{gathered}
$$

Proof of Lemma 3.3. We observe that:

$$
\begin{aligned}
\int_{0}^{2 \pi} \mathrm{e}^{\mathrm{i} \theta} \cdot \nabla_{x} E\left(x-r \mathrm{e}^{\mathrm{i} \theta+\mathrm{i} \pi / 2}\right) \cdot \mathrm{d} \theta & =\int_{0}^{2 \pi} \partial_{\theta}\left[E\left(x-r \mathrm{e}^{\mathrm{i} \theta+\mathrm{i} \pi / 2}\right)\right] \mathrm{d} \theta=0 . \\
\int_{0}^{2 \pi} \mathrm{e}^{\mathrm{i} \theta} \cdot \nabla_{x} E^{\perp}\left(x-r \mathrm{e}^{\mathrm{i} \theta+\mathrm{i} \pi / 2}\right) \cdot \mathrm{e}^{\mathrm{i} \theta} \mathrm{d} \theta & =\int_{0}^{2 \pi} \partial_{\theta}\left[E^{\perp}\left(x-r \mathrm{e}^{\mathrm{i} \theta+\mathrm{i} \pi / 2}\right)\right] \cdot \mathrm{e}^{\mathrm{i} \theta} \mathrm{d} \theta \\
& =-\int_{0}^{2 \pi}\left[E^{\perp}\left(x-r \mathrm{e}^{\mathrm{i} \theta+\mathrm{i} \pi / 2}\right)\right] \cdot \partial_{\theta} \mathrm{e}^{\mathrm{i} \theta} \mathrm{d} \theta \\
& =\int_{0}^{2 \pi} \sin \theta E_{2}\left(x-r \mathrm{e}^{\mathrm{i} \theta+\mathrm{i} \pi / 2}\right)+\cos \theta E_{1}\left(x-r \mathrm{e}^{\mathrm{i} \theta+\mathrm{i} \pi / 2}\right) \mathrm{d} \theta \\
& =\int_{0}^{2 \pi} \partial_{\theta}\left[V\left(x-r \mathrm{e}^{\mathrm{i} \theta+\mathrm{i} \pi / 2}\right)\right] \mathrm{d} \theta=0 .
\end{aligned}
$$

Proof of Theorem 3.1. To prove this result, we can closely follow the proof in [9], except for some new terms involving the polarization force and drift.

\footnotetext{
${ }^{3}$ However, we need a little more regularity on $E$ in order to give sense to the terms of the polarization force and drift. Nevertheless, in our approach, all terms have a clear physical interpretation, which is not the case in the approach of [9].
} 
We observe that $\operatorname{div}_{v}\left(F_{\varepsilon}\right)=0$. This entails that:

$$
\operatorname{div}_{x} F_{\varepsilon}^{\perp}\left(t, x-v^{\perp}, v\right)+\operatorname{div}_{v} F_{\varepsilon}\left(t, x-v^{\perp}, v\right)=0 .
$$

By Liouville's theorem, this means that all $L_{x, v}^{p}$ norms of $\left(g_{\varepsilon}\right)$ are conserved. Thus, up to a subsequence, $g_{\varepsilon}$ admits a weak limit in the sense of distributions, denoted by $g$, that we shall characterize now.

Multiplying (3.3) by $\varepsilon$ and passing to the limit $\varepsilon \rightarrow 0$, we easily get:

$$
v^{\perp} \cdot \nabla_{v} g=0 .
$$

This means that $g$ only depends on the modulus of the velocity variable, and not on the angle.

As in $[9,10]$, the idea is now to consider smooth test functions $\Psi(t, x, r)$ with a radial dependance in the velocity variable. We test the Vlasov equation against such a function, compactly supported in $\mathbb{R}_{t}^{+} \times \mathbb{R}_{x}^{2} \times \mathbb{R}_{r}^{+}$.

$$
\begin{aligned}
\int g_{\varepsilon}\left(\partial_{t} \Psi\right. & +E^{\perp}\left(t, x-v^{\perp}\right) \cdot \nabla_{x} \Psi+F_{\varepsilon}^{\perp}\left(t, x-v^{\perp}, v\right) \cdot \nabla_{x} \Psi \\
& \left.\quad+F_{\varepsilon}\left(t, x-v^{\perp}, v\right) \cdot \nabla_{v} \Psi+v^{\perp} \cdot \nabla_{v} \Psi\right) \mathrm{d} t \mathrm{~d} x \mathrm{~d} v=-\int g_{0, \varepsilon} \Psi \mathrm{d} x \mathrm{~d} v .
\end{aligned}
$$

In the following, we denote $v=r \mathrm{e}^{\mathrm{i} \theta}$. We first have, since $\Psi$ and $g$ do not depend on $\theta$, as $\varepsilon \rightarrow 0$ :

$$
\begin{aligned}
& \int g_{\varepsilon}\left(\partial_{t} \Psi+E^{\perp}\left(t, x-v^{\perp}\right) \cdot \nabla_{x} \Psi\right) \mathrm{d} t \mathrm{~d} x \mathrm{~d} v \\
& \rightarrow 2 \pi \int g \partial_{t} \Psi r \mathrm{~d} r \mathrm{~d} t \mathrm{~d} x+\iint_{0}^{2 \pi} E^{\perp}\left(t, x-|v| \mathrm{e}^{\mathrm{i} \theta+\mathrm{i} \pi / 2}\right) \mathrm{d} \theta \cdot \nabla_{x} \Psi g r \mathrm{~d} r \mathrm{~d} t \mathrm{~d} x .
\end{aligned}
$$

We also observe that $v^{\perp} \cdot \nabla_{v} \Psi=0$. Furthermore, in $L_{t}^{\infty} L_{x}^{2}$, both terms $\varepsilon \partial_{t} E, \varepsilon E^{\perp} \cdot \nabla_{x} E^{\perp}$ vanish in the limit $\varepsilon \rightarrow 0$.

Passing to the limit $\varepsilon \rightarrow 0$, we have

$$
\int g_{\varepsilon}\left(v \cdot \nabla_{x} E^{\perp}\left(t, x-v^{\perp}\right)\right) \cdot \nabla_{v} \Psi \mathrm{d} t \mathrm{~d} x \mathrm{~d} v \rightarrow \int g\left(v \cdot \nabla_{x} E^{\perp}\left(t, x-v^{\perp}\right)\right) \cdot \nabla_{v} \Psi \mathrm{d} t \mathrm{~d} x \mathrm{~d} v .
$$

We recall that $g$ and $\Psi$ have only a radial dependance in the velocity variable. We have:

$$
\int g\left(v \cdot \nabla_{x} E^{\perp}\left(t, x-v^{\perp}\right)\right) \cdot \nabla_{v} \Psi \mathrm{d} v=\int\left(\int\left(\mathrm{e}^{\mathrm{i} \theta} \cdot \nabla_{x} E^{\perp}\left(t, x-r \mathrm{e}^{\mathrm{i} \theta+\mathrm{i} \pi / 2}\right)\right) \cdot \mathrm{e}^{\mathrm{i} \theta} \mathrm{d} \theta\right) r g \partial_{r} \Psi \mathrm{d} r .
$$

Likewise, we have:

$$
\begin{aligned}
\int g_{\varepsilon}\left(v \cdot \nabla_{x} E\left(t, x-v^{\perp}\right)\right) \cdot \nabla_{x} \Psi \mathrm{d} t \mathrm{~d} x \mathrm{~d} v & \rightarrow \int g\left(v \cdot \nabla_{x} E\left(t, x-v^{\perp}\right)\right) \cdot \nabla_{x} \Psi \mathrm{d} t \mathrm{~d} x \mathrm{~d} v \\
& =\int g\left(\int \mathrm{e}^{\mathrm{i} \theta} \cdot \nabla_{x} E\left(t, x-r \mathrm{e}^{\mathrm{i} \theta+\mathrm{i} \pi / 2}\right) \mathrm{d} \theta\right) \cdot \nabla_{x} \Psi r^{2} \mathrm{~d} t \mathrm{~d} x \mathrm{~d} r .
\end{aligned}
$$

We finally use Lemma 3.3 to prove that the contributions of both of these terms vanish.

Concerning the initial data, we get:

$$
\int g_{0, \varepsilon} \Psi \mathrm{d} x \mathrm{~d} v \rightarrow \int\left(\int g_{0} \mathrm{~d} \theta\right) r \mathrm{~d} x \mathrm{~d} r .
$$

Gathering all pieces together, this proves the Theorem. 
Remark 3.4. Let us now assume that $E_{\varepsilon}$ is an external electric field, with some time oscillations of frequency $\frac{1}{\varepsilon}$. Then we can easily see that the polarization terms do not vanish in the limit $\varepsilon \rightarrow 0$. For instance we may consider:

$$
E_{\varepsilon}=\left(1-\sin \frac{t}{\varepsilon}\right) e_{2}
$$

One can observe that $E_{\varepsilon}$ is uniformly in $L_{t}^{\infty} H_{x}^{s}$ for any $s \in \mathbb{R}$. For the initial data, we take $g_{\varepsilon, 0}=g_{0}$ in $L_{v}^{\infty}$ which does not depend on $x$. Then the polarization force is given by $F_{\varepsilon}=\cos \frac{t}{\varepsilon} e_{1} \neq 0$ and (3.3) reads:

$$
\partial_{t} g_{\varepsilon}+\cos \frac{t}{\varepsilon} \partial_{v_{1}} g_{\varepsilon}+\frac{v^{\perp}}{\varepsilon} \cdot \nabla_{v} g_{\varepsilon}=0
$$

Setting $h_{\varepsilon}(t, x, v):=g_{\varepsilon}(t, x, R(-t / \varepsilon) v)$, one can show that $h_{\varepsilon}$ satisfies the equation:

$$
\partial_{t} h_{\varepsilon}+\left(\begin{array}{c}
\cos ^{2} \frac{t}{\varepsilon} \\
\frac{1}{2} \sin 2 \frac{t}{\varepsilon}
\end{array}\right) \cdot \nabla_{v} h_{\varepsilon}=0
$$

Denoting by $h$ a weak limit in $L_{t, x, v}^{\infty}$ of $h_{\varepsilon}$, one can show by some elementary computations that $h$ satisfies the limit equation:

$$
\partial_{t} h+\frac{1}{2} \partial_{v_{1}} h=0
$$

The acceleration term $\frac{1}{2} \partial_{v_{1}}$ stems from the polarization force.

\subsection{Analysis of the limit in the Poisson case}

We now investigate system (3.6) coupled with the Poisson equation, in which case the coupled system reads:

$$
\left\{\begin{array}{l}
\partial_{t} h_{\varepsilon}+E_{\varepsilon}^{\perp}(t, x+\mathcal{R}(-t / \varepsilon) v) \cdot \nabla_{x} h_{\varepsilon}+\mathcal{R}(t / \varepsilon) F_{\varepsilon}(t, x+\mathcal{R}(-t / \varepsilon) v, R(-t / \varepsilon) v) \cdot \nabla_{x} h_{\varepsilon} \\
+R(t / \varepsilon) F_{\varepsilon}(t, x+\mathcal{R}(-t / \varepsilon) v, R(-t / \varepsilon) v) \cdot \nabla_{v} h_{\varepsilon}=0, \\
E_{\varepsilon}=-\nabla_{x} \Phi_{\varepsilon}, \\
-\Delta_{x} \Phi_{\varepsilon}=\int h_{\varepsilon}(t, x+\mathcal{R}(t / \varepsilon) v, R(t / \varepsilon) v) \mathrm{d} v-1,
\end{array}\right.
$$

with $F_{\varepsilon}=-\varepsilon \partial_{t} E_{\varepsilon}^{\perp}-v+\varepsilon E_{\varepsilon}^{\perp} \cdot \nabla_{x}\left(E_{\varepsilon}^{\perp}\right)$.

Before stating the proposition, we gather in the following lemma some useful uniform estimates for the solutions $\left(f_{\varepsilon}\right)$ of $(1.6)$ and the solutions $\left(h_{\varepsilon}\right)$ of (3.12). At fist, let us give a definition which will be helpful to state some of the results.

Definition 3.5. Let $u \in \mathcal{D}^{\prime}\left(\mathbb{T}_{x}^{2} \times \mathbb{R}_{v}^{2}\right)$. Let $s \in \mathbb{R}, p \in[1,+\infty]$.

We say that $u \in W_{x, v, l o c}^{s, p}$ if for any function $\xi$ in $\mathcal{D}\left(\mathbb{T}_{x}^{2} \times \mathbb{R}_{v}^{2}\right), \xi u \in W_{x, v}^{s, p}\left(\mathbb{T}_{x}^{2} \times \mathbb{R}_{v}^{2}\right)\left(\right.$ where $W_{x, v}^{s, p}\left(\mathbb{T}_{x}^{2} \times \mathbb{R}_{v}^{2}\right)$ is the usual Sobolev space).

\section{Lemma 3.6.}

(1) The energy defined by the functional:

$$
\mathcal{E}_{\varepsilon}(t)=\int f_{\varepsilon}|v|^{2} \mathrm{~d} v \mathrm{~d} x+\varepsilon \int\left|\nabla_{x} V_{\varepsilon}\right|^{2} \mathrm{~d} x
$$

where $f_{\varepsilon}$ is solution to (1.6), is non-increasing.

All $L^{p}$ norms of $f_{\varepsilon}$ are conserved:

$$
\left\|f_{\varepsilon}\right\|_{L_{t}^{\infty} L_{x, v}^{p}} \leq\left\|f_{0, \varepsilon}\right\|_{L_{x, v}^{p}} .
$$

(2) The charge $\rho_{\varepsilon}:=\int f_{\varepsilon} \mathrm{d} v$ is uniformly bounded in $L_{t}^{\infty} L_{x}^{3 / 2}$. The current $J_{\varepsilon}:=\int f_{\varepsilon} v \mathrm{~d} v$ is uniformly bounded in $L_{t}^{\infty} L_{x}^{5 / 4}$. 
(3) Let

$$
S_{\varepsilon}=\mathcal{R}(t / \varepsilon) F_{\varepsilon}(t, x+\mathcal{R}(-t / \varepsilon) v, R(-t / \varepsilon) v) \cdot \nabla_{x} h_{\varepsilon}+R(t / \varepsilon) F_{\varepsilon}(t, x+\mathcal{R}(-t / \varepsilon) v, R(-t / \varepsilon) v) \cdot \nabla_{v} h_{\varepsilon} .
$$

Then $S_{\varepsilon}$ is uniformly bounded in $L_{t}^{\infty} W_{x, v, l o c}^{-1,6 / 5}$.

(4) The sequence $h_{\varepsilon}$ is strongly relatively compact in $L_{t, l o c}^{\infty} W_{x, v, l o c}^{-1,3 / 2}$.

Sketch of proof.

1. The property of the energy follows from an explicit computation of $\frac{\mathrm{d} \mathcal{E}_{\varepsilon}}{\mathrm{d} t}$. We refer for instance to [6] or [12]. The conservation of $L^{p}$ norms comes from Liouville's theorem (since the force field is divergence free in $v$ ).

2. The estimates come from a by now very classical principle of real interpolation. It relies on the facts that $f_{\varepsilon}$ is in $L_{t, x, v}^{\infty}$ uniformly in $\varepsilon$ and $f_{\varepsilon}|v|^{2}$ is in $L_{t}^{\infty} L_{x, v}^{1}$ uniformly in $\varepsilon$. The principle is to decompose the velocity space in two parts:

$$
\int f_{\varepsilon} \mathrm{d} v=\int_{|v| \leq R} f_{\varepsilon} \mathrm{d} v+\int_{|v|>R} f_{\varepsilon} \mathrm{d} v
$$

and optimize in $R$.

3. We first recall that:

$$
F_{\varepsilon}=-\varepsilon \partial_{t} E_{\varepsilon}^{\perp}-v \cdot \nabla_{x}\left(E_{\varepsilon}^{\perp}\right)+\varepsilon E_{\varepsilon}^{\perp} \cdot \nabla_{x}\left(E_{\varepsilon}^{\perp}\right) .
$$

Therefore, one can readily check that $\operatorname{div}_{v} F_{\varepsilon}=0$, so that after the change of variables, using the chain rule, we have:

$$
\operatorname{div}_{x}\left[\mathcal{R}(t / \varepsilon) F_{\varepsilon}(t, x+\mathcal{R}(-t / \varepsilon) v, R(-t / \varepsilon) v)\right]+\operatorname{div}_{v}\left[R(t / \varepsilon) F_{\varepsilon}(t, x+\mathcal{R}(-t / \varepsilon) v, R(-t / \varepsilon) v)\right]=0 .
$$

Therefore we can deduce that $S_{\varepsilon}$ can be rewritten as:

$$
\operatorname{div}_{x}\left[\mathcal{R}(t / \varepsilon) F_{\varepsilon}(t, x+\mathcal{R}(-t / \varepsilon) v, R(-t / \varepsilon) v) h_{\varepsilon}\right]+\operatorname{div}_{v}\left[R(t / \varepsilon) F_{\varepsilon}(t, x+\mathcal{R}(-t / \varepsilon) v, R(-t / \varepsilon) v) h_{\varepsilon}\right] .
$$

We claim that $F_{\varepsilon} h_{\varepsilon} \in L_{t}^{\infty}\left(L_{x, v, l o c}^{6 / 5}\right)$ uniformly in $\varepsilon$. First, using the local conservation of charge (obtained by integrating the transport equation in (1.6)):

$$
\partial_{t} \int f_{\varepsilon} \mathrm{d} v+\frac{1}{\varepsilon} \operatorname{div}_{x} \int f_{\varepsilon} v \mathrm{~d} v=0
$$

and derivating with respect to time the Poisson equation in (1.6):

$$
-\Delta_{x} \partial_{t} V_{\varepsilon}=\partial_{t} \int f_{\varepsilon} \mathrm{d} v
$$

we obtain:

$$
\varepsilon \partial_{t} E_{\varepsilon}=\nabla_{x} \Delta_{x}^{-1} \operatorname{div}_{x} \int f_{\varepsilon} v \mathrm{~d} v .
$$

As a result, using point 2 of the lemma and by elliptic regularity, we have:

$$
-\varepsilon \partial_{t} E_{\varepsilon}^{\perp} \in L_{t}^{\infty} L_{x}^{5 / 4}
$$

uniformly in $\varepsilon$.

By point 2 of the lemma, $\rho_{\varepsilon} \in L_{t}^{\infty} L_{x}^{3 / 2}$. Consequently, by elliptic regularity, we get $E_{\varepsilon} \in L_{t}^{\infty}\left(W_{x}^{1,3 / 2}\right)$, and thus by Sobolev embedding in $2 D$, we have $E_{\varepsilon} \in L_{t}^{\infty}\left(L_{x}^{6}\right)$ (uniformly in $\varepsilon$ ). By Hölder's inequality, we have:

This means that $F_{\varepsilon}$ is uniformly in $L_{t}^{\infty} L_{x, v, l o c}^{6 / 5}$.

$$
E_{\varepsilon}^{\perp} \cdot \nabla_{x} E_{\varepsilon}^{\perp} \in L_{t}^{\infty} L_{x}^{6 / 5} .
$$

Since all $L^{p}$ norms of $h_{\varepsilon}$ are conserved, we have $h_{\varepsilon} \in L_{t}^{\infty} L_{x, v}^{\infty}$ uniformly in $\varepsilon$ and thus $F_{\varepsilon} h_{\varepsilon} \in L_{t}^{\infty}\left(L_{x, v, l o c}^{6 / 5}\right)$. 
4. We use the transport equation satisfied by $h_{\varepsilon}$ in (3.12) and the uniform bound on $S_{\varepsilon}$ to show that $\partial_{t} h_{\varepsilon}$ is uniformly in $L_{t}^{\infty} W_{x, v, l o c}^{-1,6 / 5}$. In addition, we have $h_{\varepsilon} \in L_{t}^{\infty} L_{x, v, l o c}^{\infty}$. Then, the compactness property relies on the Aubin-Lions compactness lemma (see for instance [6] for more details).

We now prove the following proposition:

Proposition 3.7. Let $\left(f_{\varepsilon, 0}\right)$ be a sequence of initial data uniformly bounded in $L_{x, v}^{1} \cap L_{x, v}^{\infty}$ and with uniformly bounded energy $\mathcal{E}_{\varepsilon}(0)$.

We assume that $\left(h_{\varepsilon, 0}\right)$ weakly converges in $L_{x, v}^{2}$ to some $h_{0} \in L_{x, v}^{1} \cap L_{x, v}^{2}$. Then there exists $S \in L_{t}^{\infty}\left(W_{x, v, l o c}^{-1, q}\right)$ such that $h_{\varepsilon}$ solution to (3.12) with initial data $h_{\varepsilon, 0}$ weakly converges in the sense of distributions, up to $a$ subsequence, to $h$, solution to the non linear transport equation:

$$
\left\{\begin{array}{l}
\partial_{t} h+\frac{1}{2 \pi} \int_{0}^{2 \pi} E^{\perp}(t, \tau, x+\mathcal{R}(-\tau) v) \mathrm{d} \tau \cdot \nabla_{x} h=-S \\
E=-\nabla_{x} \Phi \\
-\Delta_{x} \Phi=\int h(t, x+\mathcal{R}(\tau) v, R(\tau) v) \mathrm{d} v-1 \\
h_{\mid t=0}=h_{0} .
\end{array}\right.
$$

For the moment, this result is not very satisfactory since we are not able to compute or characterize the "source" $S$, which comes from the polarization drift and force that we have introduced earlier. In the next subsection, we will nevertheless explain how to compute $S$ (by another way).

The proof of Proposition 3.7 relies on two-scale convergence notions, that we recall for the sake of readability. This notion is useful in this context since it is more precise than weak convergence, insofar as it can capture the oscillating behaviour of the sequence of the functions under study.

Definition 3.8. Let $X$ be a separable Banach space, $X^{\prime}$ be its topological dual space and (.,.) the duality bracket between $X^{\prime}$ and $X$. For all $\alpha>0$, denote by $\mathcal{C}_{\alpha}(\mathbb{R}, X)$ (respectively $L_{\alpha}^{q^{\prime}}\left(\mathbb{R} ; X^{\prime}\right)$ ) the space of $\alpha$-periodic continuous (respectively $L^{q^{\prime}}$ ) functions on $\mathbb{R}$ with values in $X$. Let $q \in[1 ; \infty[$.

Given a sequence $\left(u_{\varepsilon}\right)$ of functions belonging to the space $L^{q^{\prime}}\left(0, t ; X^{\prime}\right)$ and a function $U^{0}(t, \theta) \in$ $L^{q^{\prime}}\left(0, T ; L_{\alpha}^{q^{\prime}}\left(\mathbb{R} ; X^{\prime}\right)\right)$ we say that

$$
u_{\varepsilon} 2 \text {-scale converges to } U^{0}
$$

if for any function $\Psi \in L^{q}\left(0, T ; \mathcal{C}_{\alpha}(\mathbb{R}, X)\right)$ we have:

$$
\lim _{\varepsilon \rightarrow 0} \int_{0}^{T}\left(u_{\varepsilon}(t), \Psi\left(t, \frac{t}{\varepsilon}\right)\right) \mathrm{d} t=\frac{1}{\alpha} \int_{0}^{T} \int_{0}^{\alpha}\left(U^{0}(t, \tau), \Psi(t, \tau)\right) \mathrm{d} \tau \mathrm{d} t
$$

The main theorem we use is the following, due to Nguetseng [17] and Allaire [1].

Theorem 3.9 (Nguetseng and Allaire). Given a sequence $\left(u_{\varepsilon}\right)$ bounded in $L^{q^{\prime}}\left(0, t ; X^{\prime}\right)$, there exists for all $\alpha>0$ a function $U_{\alpha}^{0} \in L^{q^{\prime}}\left(0, T ; L_{\alpha}^{q^{\prime}}\left(\mathbb{R} ; X^{\prime}\right)\right)$ such that up to a subsequence,

$$
u_{\varepsilon} \text { 2-scale converges to } U_{\alpha}^{0} \text {. }
$$

The profile $U_{\alpha}^{0}$ is called the $\alpha$-periodic two scale limit of $u_{\varepsilon}$ and the link between $U_{\alpha}^{0}$ and the weak-* limit $u$ of $u_{\varepsilon}$ is given by:

$$
\frac{1}{\alpha} \int_{0}^{\alpha} U^{0} \mathrm{~d} \tau=u
$$

Proof of Proposition 3.7. We have the following convergences in the sense of distributions:

$$
\begin{aligned}
& h_{\varepsilon} \rightarrow h, \\
& E_{\varepsilon} \rightarrow E,
\end{aligned}
$$

since these terms are uniformly bounded in some $L^{p}$ space. 
Using two-scale convergence tools and the compactness obtained in point 4 of Lemma 3.6 we can show:

$$
E_{\varepsilon}^{\perp}(t, x+\mathcal{R}(-t / \varepsilon) v) \cdot \nabla_{x} h_{\varepsilon} \rightarrow \frac{1}{2 \pi} \int_{0}^{2 \pi} E^{\perp}(t, \tau, x+\mathcal{R}(-\tau) v) \mathrm{d} \tau \cdot \nabla_{x} h .
$$

Furthermore, by point 3 of Lemma 3.6, $S_{\varepsilon}$ is uniformly bounded in $L_{t}^{\infty} W_{x, v, l o c}^{-1, q}$ and up to a subsequence it converges (in the sense of distributions) to some $S$ in $L_{t}^{\infty} W_{x, v, l o c}^{-1, q}$.

Passing to the limit $\varepsilon \rightarrow 0$ in the sense of distributions, we finally get:

$$
\partial_{t} h+\frac{1}{2 \pi} \int_{0}^{2 \pi} E^{\perp}(t, \tau, x+\mathcal{R}(-\tau) v) \mathrm{d} \tau \cdot \nabla_{x} h=-S .
$$

Finally, one can also pass to the limit in the Poisson equation, using two-scale convergence tools. We refer to $[6]$ or $[12]$ for more details.

\subsection{Relevancy and a new interpretation of Frénod and Sonnendrücker's asymptotic equation}

Let $\varepsilon>0$ and $f_{\varepsilon}$ a solution to (1.6). If we directly set:

$$
\tilde{h}_{\varepsilon}=f_{\varepsilon}(t, x+\mathcal{R}(-t / \varepsilon) v, R(-t / \varepsilon) v),
$$

we recall that in [6], it is shown that up to a subsequence, $\tilde{h}_{\varepsilon}$ converges in the sense of distributions to $\tilde{h}$ solution to:

$$
\left\{\begin{array}{l}
\partial_{t} \tilde{h}+\frac{1}{2 \pi}\left(\int_{0}^{2 \pi} \mathcal{R}(\tau) \mathcal{E}(t, \tau, x+\mathcal{R}(-\tau) v) \mathrm{d} \tau\right) \cdot \nabla_{x} \tilde{h} \\
+\frac{1}{2 \pi}\left(\int_{0}^{2 \pi} R(\tau) \mathcal{E}(t, \tau, x+\mathcal{R}(-\tau) v) \mathrm{d} \tau\right) \cdot \nabla_{v} \tilde{h}=0 \\
\mathcal{E}=-\nabla \Phi,-\Delta \Phi=\int \tilde{h}(t, x+\mathcal{R}(\tau) v, R(\tau) v) \mathrm{d} v-1, \\
\tilde{h}_{\mid t=0}=f_{0} .
\end{array}\right.
$$

We recall now that we have considered in this work the following distribution function:

$$
h_{\varepsilon}=f_{\varepsilon}^{\prime}(t, x+\mathcal{R}(-t / \varepsilon) v, R(-t / \varepsilon) v) .
$$

In Proposition 3.7, we have proved that any weak limit $h$ satisfies (3.15). In the definition (3.1) of $f_{\varepsilon}^{\prime}$, we observe that because of the conservation of energy in Lemma 3.6, $\sqrt{\varepsilon} E_{\varepsilon}$ is bounded in $L_{t}^{\infty} L_{x}^{2}$ and therefore $\varepsilon E_{\varepsilon}^{\perp}$ vanishes as $\varepsilon$ goes to 0 , so we should have $h=\tilde{h}$. This is the matter of the next Theorem, thanks to which we are able to compute $S$.

Theorem 3.10. Let $\left(f_{0, \varepsilon}\right)$ be a sequence of non-negative initial data, uniformly bounded in $L_{x, v}^{1} \cap L_{x, v}^{\infty}$.

(1) Up to a subsequence, we have the convergence, in the sense of distributions:

$$
h_{\varepsilon}-\tilde{h}_{\varepsilon} \rightarrow 0 .
$$

(2) Assume that the whole sequence $f_{0, \varepsilon}$ converges to some $f_{0}$ satisfying:

$$
\begin{array}{r}
f_{0} \in W_{x, v}^{1,1}, \\
\left\|\left(1+|v|^{4}\right) f_{0}\right\|_{L_{x, v}^{\infty}}<\infty, \\
\left\|\left(1+|v|^{4}\right) \nabla_{x, v} f_{0}\right\|_{L_{x, v}^{\infty}}<\infty .
\end{array}
$$


Then (3.20) holds without extraction. Furthermore, with the notations of Proposition 3.7, we have:

$$
\begin{aligned}
S= & \frac{1}{2 \pi}\left(\int_{0}^{2 \pi}\left(\begin{array}{cc}
\sin \tau & -\cos \tau \\
\cos \tau & \sin \tau
\end{array}\right) \mathcal{E}(t, \tau, x+\mathcal{R}(-\tau) v) \mathrm{d} \tau\right) \cdot \nabla_{x} h \\
& +\frac{1}{2 \pi}\left(\int_{0}^{2 \pi} R(\tau) \mathcal{E}(t, \tau, x+\mathcal{R}(-\tau) v) \mathrm{d} \tau\right) \cdot \nabla_{v} h .
\end{aligned}
$$

This theorem rigorously justifies our previous heuristic statements: equation (1.8) actually implicitly takes into account the effects of the polarization drift (and actually even more, see Sect. 2). In other words, the formerly mysterious terms (1.13) are a direct consequence of polarization effects.

Proof.

1. Let $\Psi(t, x, v)$ be a smooth test function in $\mathcal{D}\left(\mathbb{R}_{t}^{+} \times \mathbb{T}_{x}^{2} \times \mathbb{R}_{v}^{2}\right)$. We can evaluate, by definition of $h_{\varepsilon}$ (in (3.19)) and $\tilde{h}_{\varepsilon}($ in $(3.18))$ :

$$
\begin{aligned}
\int & \left(h_{\varepsilon}(t, x, v)-\tilde{h}_{\varepsilon}(t, x, v)\right) \Psi(t, x, v) \mathrm{d} t \mathrm{~d} x \mathrm{~d} v \\
= & \int\left(f_{\varepsilon}^{\prime}(t, x+\mathcal{R}(-t / \varepsilon) v, R(-t / \varepsilon) v)-f_{\varepsilon}(t, x+\mathcal{R}(-t / \varepsilon) v, R(-t / \varepsilon) v)\right) \Psi \mathrm{d} t \mathrm{~d} x \mathrm{~d} v \\
= & \int\left(f_{\varepsilon}\left(t, x+\mathcal{R}(-t / \varepsilon) v, R(-t / \varepsilon) v+\varepsilon E_{\varepsilon}^{\perp}(t, x+\mathcal{R}(-t / \varepsilon) v)\right)\right. \\
& \left.-f_{\varepsilon}(t, x+\mathcal{R}(-t / \varepsilon) v, R(-t / \varepsilon) v)\right) \Psi \mathrm{d} t \mathrm{~d} x \mathrm{~d} v \\
= & \int f_{\varepsilon}\left(t, x^{\prime}, v^{\prime}\right)\left(\Psi\left(t, x^{\prime}+\mathcal{R}(t / \varepsilon) v^{\prime}-\varepsilon \mathcal{R}(t / \varepsilon) E_{\varepsilon}^{\perp}\left(t, x^{\prime}\right), R(t / \varepsilon) v^{\prime}-\varepsilon E_{\varepsilon}^{\perp}\left(t, x^{\prime}\right)\right)\right. \\
& \left.-\Psi\left(t, x^{\prime}+\mathcal{R}(t / \varepsilon) v^{\prime}, R(t / \varepsilon) v^{\prime}\right)\right) \mathrm{d} t \mathrm{~d} x^{\prime} \mathrm{d} v^{\prime} .
\end{aligned}
$$

In the last line of this computation, we have performed the changes of variables (each has unit Jacobian):

$$
\left\{\begin{array}{l}
x^{\prime}=x+\mathcal{R}(-t / \varepsilon) v, \\
v^{\prime}=R(-t / \varepsilon) v+\varepsilon E_{\varepsilon}^{\perp}\left(t, x^{\prime}\right),
\end{array}\right.
$$

and

$$
\left\{\begin{array}{l}
x^{\prime}=x \\
v^{\prime}=R(-t / \varepsilon) v .
\end{array}\right.
$$

Hence, the following bound holds, after using Taylor's formula for $\Psi$ and Cauchy-Schwarz inequality:

$$
\left|\int\left(h_{\varepsilon}(t, x, v)-\tilde{h}_{\varepsilon}(t, x, v)\right) \Psi(t, x, v) \mathrm{d} t \mathrm{~d} x \mathrm{~d} v\right| \leq \sqrt{\varepsilon} C(\Psi)\left\|f_{\varepsilon}\right\|_{L_{t}^{\infty} L_{x, v}^{2}}\left\|\sqrt{\varepsilon} E_{\varepsilon}\right\|_{L_{t}^{\infty} L_{x}^{2}}\|\Psi\|_{W_{t, x, v}^{1, \infty}} .
$$

Using the energy estimate (point 1 of Lem. 3.6), we recall that $\sqrt{\varepsilon} E_{\varepsilon}$ is uniformly bounded in $L_{t}^{\infty} L_{x}^{2}$, and therefore this proves our claim.

2. Under the additional smoothness assumption (3.21), it was observed in a Remark of [12] that there is uniqueness of a solution $\tilde{h}$ to (1.8). Such a statement is classical for the "usual" Vlasov-Poisson system. For the system under study, the result is very similar. This relies on some fixed point arguments in the spirit of [18] or [4]. This implies that $\tilde{h}_{\varepsilon} \rightarrow \tilde{h}$ without having to take a subsequence. This means also that the whole sequence $h_{\varepsilon}$ converges in the sense of distributions to $\tilde{h}$.

In Proposition 3.7, we have at the same time proved that $h_{\varepsilon}$ (up to a subsequence) converges in the sense of distributions to $h$, solution to (3.15). Consequently, we infer that $\tilde{h}=h$, which proves our claim. 
Remark 3.11. We believe it is possible to deal with weaker assumptions than (3.21), maybe if we use the approach due to Lions and Perhame [15] for the uniqueness problem for the Vlasov-Poisson system.

Acknowledgements. I wish to thank Emmanuel Frénod and Laure Saint-Raymond for their comments on a first version of the paper.

\section{Appendix A. Finite Larmor Radius Regime}

The scaling is by now classical since it was studied in many papers, see for instance $[6,9,12,13]$. We recall the main lines in the $2 D$ setting, for the sake of completeness.

We first introduce the dimensionless variables and quantities:

$$
\begin{gathered}
\tilde{x}=\frac{x}{L}, \quad \tilde{t}=\frac{t}{\tau}, \quad \tilde{v}=\frac{v}{v_{t h}} \\
f(t, x, v)=\bar{f} \tilde{f}(\tilde{t}, \tilde{x}, \tilde{v}) \quad V(t, x, x)=\bar{V} \tilde{V}(\tilde{t}, \tilde{x}, \tilde{x}) \quad E(t, x)=\bar{E} \tilde{E}(\tilde{t}, \tilde{x}) .
\end{gathered}
$$

This yields the Vlasov-Poisson system:

$$
\left\{\begin{array}{l}
\partial_{\tilde{t}} \tilde{f}_{\varepsilon}+\frac{v_{t h} \tau}{L} \tilde{v} \cdot \nabla_{\tilde{x}} \tilde{f}_{\varepsilon}+\left(\frac{e \bar{E} \tau}{m v_{t h}} \tilde{E}_{\varepsilon}+\frac{e \bar{B}}{m} \tau \tilde{v}^{\perp}\right) \cdot \nabla_{\tilde{v}} \tilde{f}_{\varepsilon}=0 \\
\bar{E} \tilde{E}_{\varepsilon}=-\frac{1}{L} \nabla_{\tilde{x}} \tilde{V}_{\varepsilon} \\
-\frac{\varepsilon_{0} \bar{V}}{L^{2}} \Delta_{\tilde{x}} \tilde{V}_{\varepsilon}=e \bar{f} v_{t h}^{2}\left(\int \tilde{f}_{\varepsilon} d \tilde{v}-1\right) \\
\tilde{f}_{\varepsilon, \mid \tilde{t}=0}=\tilde{f}_{0, \varepsilon}, \quad \bar{f} L^{2} v_{t h}^{2} \int \tilde{f}_{0, \varepsilon} \mathrm{d} \tilde{v} \mathrm{~d} \tilde{x}=1 .
\end{array}\right.
$$

In order to have normalized distributions, it is first natural to set $\bar{f} L_{\perp}^{2} L_{\|} v_{t h}^{3}=1$.

As in Section 2, we set $\Omega=\frac{e \bar{B}}{m}$ : this is the cyclotron frequency (also referred to as the gyrofrequency) We also consider the so-called electron Larmor radius (or electron gyroradius) $r_{L}$ defined by:

$$
r_{L}=\frac{v_{t h}}{\Omega}=\frac{m v_{t h}}{e \bar{B}} .
$$

As explained in Section 2, this can be physically understood as the typical radius of the helix around axis $e_{\|}$ described by the particles, due to the intense magnetic field. We also introduce the so-called Debye length:

$$
\lambda_{D}=\frac{\varepsilon_{0} \bar{V}}{e \bar{f} v_{t h}^{3}},
$$

which is interpreted as the typical length above which the plasma can be interpreted as being neutral.

The Vlasov equation now reads:

$$
\partial_{\tilde{t}} \tilde{f}_{\varepsilon}+\frac{r_{L}}{L} \Omega \tau \tilde{v} \cdot \nabla_{\tilde{x}} \tilde{f}_{\varepsilon}+\left(\frac{\bar{E}}{\bar{B} v_{t h}} \Omega \tau \tilde{E}_{\varepsilon}+\Omega \tau \tilde{v}^{\perp}\right) \cdot \nabla_{\tilde{v}} \tilde{f}_{\varepsilon}=0 .
$$

The strong magnetic field ordering (roughly speaking it corresponds to $\bar{B} \rightarrow+\infty$ ) consists in taking:

$$
\Omega \tau=\frac{1}{\varepsilon}, \quad \frac{\bar{E}}{\bar{B} v_{t h}}=\varepsilon .
$$

The spatial scaling we perform is the so-called finite Larmor radius scaling: basically the idea is to consider the typical spatial length $L$ with the same order as the so-called electron Larmor radius.

$$
\frac{r_{L}}{L}=1
$$


The quasineutral ordering we adopt is the following:

$$
\frac{\lambda_{D}}{L}=1 / \sqrt{\varepsilon}
$$

After straightforward computations, we get the following Vlasov-Poisson system in dimensionless form, for $t \geq 0, x \in \mathbb{T}^{2}, v \in \mathbb{R}^{2}$ :

$$
\left\{\begin{array}{l}
\partial_{t} f_{\varepsilon}+\frac{v}{\varepsilon} \cdot \nabla_{x} f_{\varepsilon}+\left(E_{\varepsilon}+\frac{v^{\perp}}{\varepsilon}\right) \cdot \nabla_{v} f_{\varepsilon}=0 \\
E_{\varepsilon}=-\nabla_{x} V_{\varepsilon} \\
-\Delta_{x} V_{\varepsilon}=\int f_{\varepsilon} \mathrm{d} v-\int f_{\varepsilon} \mathrm{d} v \mathrm{~d} x \\
f_{\varepsilon, t=0}=f_{\varepsilon, 0}
\end{array}\right.
$$

\section{Appendix B. Formal Derivation of A NEW GYRokinetic Model}

We consider that our study mainly indicates that two-scale numerical methods should be developed in order to accurately describe turbulence in plasmas. Lately, there have been improvements and recent advances for the numerical simulation of two-scale models. We refer to the works of Frénod et al. [7,8], Mouton [16] and references therein. Nevertheless for the time being, such models remain difficult to simulate and are expensive, due to the introduction of a new variable (namely the fast time variable $\tau$ ).

In this appendix, the aim is to formally derive a new model which may be easier to handle in numerical simulations than (1.8), but which would keep some polarization effects. We are first able to compute the weak limit of $F_{\varepsilon}$ :

Proposition B.1. In the Vlasov-Poisson case of (3.12), the polarization force $F_{\varepsilon}$ converges in the sense of distributions to:

$$
F:=\nabla^{\perp} \Delta^{-1} \operatorname{div}\left(\int f^{\prime}(t, x, v) v\right)-v \cdot \nabla_{x} E^{\perp},
$$

where $f^{\prime}$ is a weak limit of $f_{\varepsilon}^{\prime}$ and $E$ a weak limit of $E_{\varepsilon}$.

Proof. We have $F_{\varepsilon}=-\varepsilon \partial_{t} E_{\varepsilon}^{\perp}-\left(v+\varepsilon E_{\varepsilon}^{\perp}\right) \cdot \nabla_{x}\left(E_{\varepsilon}^{\perp}\right)$. Recalling (3.14), we can compute:

$$
\begin{aligned}
-\varepsilon \partial_{t} E_{\varepsilon}^{\perp} & =\nabla^{\perp} \Delta^{-1} \operatorname{div}_{x} \int f_{\varepsilon}(t, x, v) v \mathrm{~d} v \\
& =\nabla^{\perp} \Delta^{-1} \operatorname{div}\left(\int f_{\varepsilon}^{\prime}(t, x, v) v \mathrm{~d} v+\varepsilon E_{\varepsilon}^{\perp} \int f_{\varepsilon}^{\prime}(t, x, v) \mathrm{d} v\right) \\
& =\nabla^{\perp} \Delta^{-1} \operatorname{div}\left(\int f_{\varepsilon}^{\prime}(t, x, v) v \mathrm{~d} v+\varepsilon E_{\varepsilon}^{\perp} \rho_{\varepsilon}\right) .
\end{aligned}
$$

Now we can observe that:

$$
\nabla^{\perp} \Delta^{-1} \operatorname{div} E_{\varepsilon}^{\perp} \rho_{\varepsilon}=E_{\varepsilon}^{\perp} \cdot \nabla_{x} E_{\varepsilon}^{\perp} .
$$

Indeed, taking the curl of each quantity, this is equivalent to prove that:

$$
\operatorname{div} E_{\varepsilon}^{\perp} \rho_{\varepsilon}=\operatorname{curl}\left(E_{\varepsilon}^{\perp} \cdot \nabla_{x} E_{\varepsilon}^{\perp}\right) .
$$

Recalling the Poisson equation:

$$
\rho_{\varepsilon}-1=\operatorname{div} E_{\varepsilon},
$$

one can easily check that each quantity is equal to:

$$
\partial_{1}\left(E_{2} \partial_{2} E_{2}+E_{2} \partial_{1} E_{1}\right)-\partial_{2}\left(E_{1} \partial_{2} E_{2}+E_{1} \partial_{1} E_{1}\right) .
$$


Finally, we have:

$$
F_{\varepsilon}=\nabla^{\perp} \Delta^{-1} \operatorname{div}\left(\int f_{\varepsilon}^{\prime}(t, x, v) v \mathrm{~d} v\right)-v \cdot \nabla_{x} E_{\varepsilon} .
$$

In the sense of distributions we obtain:

$$
v \cdot \nabla_{x}\left(E_{\varepsilon}^{\perp}\right) \rightarrow v \cdot \nabla_{x} E^{\perp} .
$$

Denote by $f^{\prime}$ a weak limit of $f_{\varepsilon}^{\prime}$. Then we have in the distributional sense:

$$
\int f_{\varepsilon}^{\prime}(t, x, v) v \mathrm{~d} v \rightarrow \int f^{\prime}(t, x, v) v \mathrm{~d} v .
$$

Finally we have shown that $F_{\varepsilon}$ weakly converges to $F$ defined by:

$$
F=\nabla^{\perp} \Delta^{-1} \operatorname{div}\left(\int f^{\prime}(t, x, v) v \mathrm{~d} v\right)-v \cdot \nabla_{x} E^{\perp} .
$$

Following the computations of Theorem 3.1 and Proposition B.1, we can pass formally to the limit in (3.3) and thus we can propose the following new model which displays some effects of the polarization drift:

$$
\left\{\begin{array}{l}
\partial_{t} g+\frac{1}{2 \pi} \int_{0}^{2 \pi} E^{\perp}\left(t, x-r \mathrm{e}^{\mathrm{i} \theta+\mathrm{i} \pi / 2}\right) \mathrm{d} \theta \cdot \nabla_{x} g \quad t \geq 0, x \in \mathbb{T}^{2}, r \geq 0 \\
-\frac{1}{2 \pi} \int_{0}^{2 \pi} \tilde{J}\left(t, x-r \mathrm{e}^{\mathrm{i} \theta+\mathrm{i} \pi / 2}\right) \mathrm{d} \theta \cdot \nabla_{x} g+\frac{1}{2 \pi} \int_{0}^{2 \pi} \mathrm{e}^{\mathrm{i} \theta} \cdot \tilde{J}^{\perp}\left(t, x-r \mathrm{e}^{\mathrm{i} \theta+\mathrm{i} \pi / 2}\right) \mathrm{d} \theta \partial_{r} g=0 . \\
E=-\nabla_{x} \Phi \\
-\Delta_{x} \Phi=\frac{1}{2 \pi} \int_{0}^{2 \pi} \int_{\mathbb{R}^{+}} g\left(t, x-r \mathrm{e}^{\mathrm{i} \theta+\mathrm{i} \pi / 2}, r\right) r \mathrm{~d} r \mathrm{~d} \theta-1 \\
\tilde{J}=\nabla \Delta^{-1} \operatorname{div}\left(\frac{1}{2 \pi} \int_{0}^{2 \pi} \int_{\mathbb{R}^{+}} g\left(t, x-r \mathrm{e}^{\mathrm{i} \theta+\mathrm{i} \pi / 2}, r\right) r^{2} \mathrm{e}^{\mathrm{i} \theta} \mathrm{d} r \mathrm{~d} \theta\right) .
\end{array}\right.
$$

However, it seems really difficult to study this system from the mathematical point of view, since the field $\tilde{J}$ only a priori belongs to some Lebesgue space, so there is a lack of regularity for this field. We can not even use an averaging lemma to overcome this feature, since the free transport operator has disappeared in the process.

\section{REFERENCES}

[1] G. Allaire, Homogenization and two-scale convergence. SIAM J. Math. Anal. XXIII (1992) 1482-1518.

[2] A.A. Arsenev, Existence in the large of a weak solution of Vlasov's system of equations. Z. Vychisl. Mat. Mat. Fiz. 15 (1975) $136-147$.

[3] M. Bostan, The Vlasov-Poisson system with strong external magnetic field. Finite Larmor radius regime. Asymptot. Anal. 61 (2009) 91-123.

[4] P. Degond, Global existence of smooth solutions for the Vlasov-Fokker-Planck equations in 1 and 2 space dimensions. Ann. Sci. École Norm. Sup. 19 (1986) 519-542.

[5] E. Frénod and A. Mouton, Two-dimensional finite Larmor radius approximation in canonical gyrokinetic coordinates. J. Pure Appl. Math.: Adv. Appl. 4 (2010) 135-166.

[6] E. Frénod and E. Sonnendrücker, The finite Larmor radius approximation. SIAM J. Math. Anal. 32 (2001) 1227-1247.

[7] E. Frénod, A. Mouton and E. Sonnendrücker, Two-scale numerical simulation of the weakly compressible 1D isentropic Euler equations. Numer. Math. 108 (2007) 263-293.

[8] E. Frénod, F. Salvarani and E. Sonnendrücker, Long time simulation of a beam in a periodic focusing channel via a two-scale PIC-method. Math. Models Methods Appl. Sci. 19 (2009) 175-197.

[9] P. Ghendrih, M. Hauray and A. Nouri, Derivation of a gyrokinetic model. Existence and uniqueness of specific stationary solutions. KRM 2 (2009) 707-725.

[10] F. Golse and L. Saint-Raymond, The Vlasov-Poisson system with strong magnetic field. J. Math. Pures Appl. 78 (1999) 791-817.

[11] V. Grandgirard et al., Global full- $f$ gyrokinetic simulations of plasma turbulence. Plasma Phys. Control. Fusion 49 (2007) 173-182. 
[12] D. Han-Kwan, The three-dimensional finite Larmor radius approximation. Asymptot. Anal. 66 (2010) 9-33.

[13] D. Han-Kwan, On the three-dimensional finite Larmor radius approximation: the case of electrons in a fixed background of ions. Submitted (2010).

[14] Z. Lin, S. Ethier, T.S. Hahm and W.M. Tang, Size scaling of turbulent transport in magnetically confined plasmas. Phys. Rev. Lett. 88 (2002) 195004-1-195004-4.

[15] P.L. Lions and B. Perthame, Propagation of moments and regularity for the three-dimensional Vlasov-Poisson system. Invent. Math. 105 (1991) 415-430.

[16] A. Mouton, Two-scale semi-Lagrangian simulation of a charged particle beam in a periodic focusing channel. KRM 2 (2009) 251-274.

[17] G. Nguetseng, A general convergence result for a functional related to the theory of homogenization. SIAM J. Math. Anal. 20 (1989) 608-623.

[18] S. Ukai and T. Okabe, On classical solutions in the large in time of two-dimensional Vlasov's equation. Osaka J. Math. 15 (1978) 245-261.

[19] J. Wesson, Tokamaks. Clarendon Press-Oxford (2004). 